J. Dairy Sci. 99:9581-9585

http://dx.doi.org/10.3168/jds.2016-11780

(C) American Dairy Science Association ${ }^{\circledR}, 2016$.

\title{
Short communication: A novel method using immunomagnetic separation with a fluorescent nanobeads lateral flow assay for the rapid detection of low-concentration Escherichia coli 0157:H7 in raw milk
}

\author{
Zhen Huang, ${ }^{*}$ Xi Cui, ${ }^{*}$ Quan-Yuan Xie, ${ }^{*}$ Dao-Feng Liu, $†$ and Wei-Hua Lai ${ }^{* 1}$ \\ *State Key Laboratory of Food Science and Technology, Nanchang University, Nanchang 330047, China \\ †Jiangxi Province Centers for Disease Control and Prevention, Nanchang 330096, China
}

\begin{abstract}
Escherichia coli O157:H7 is an important serotype of enterohemorrhagic E. coli that was first identified as a human pathogen in 1982. This pathogen causes several serious diseases. In this study, immunomagnetic separation was coupled with a fluorescent nanobeads lateral flow assay to establish a sensitive and rapid detection method for Escherichia coli O157:H7 in raw milk. The pathogen was captured from raw milk by immunomagnetic separation with immunomagnetic nanobeads and then detected using a fluorescent nanobeads lateral flow assay. A fluorescent line was formed in the test line of the test strip and quantitatively detected using a fluorescent reader. Screening times, which included immunomagnetic separation and the fluorescent nanobeads lateral flow assay, were $8,7,6$, and $5 \mathrm{~h}$ when 1 , 5, 25, and 125 cfu of E. coli O157:H7, respectively, were inoculated into $25 \mathrm{~mL}$ of raw milk. The established method could be widely applied to the rapid onsite detection of other pathogens to ensure food safety.

Key words: immunomagnetic separation, fluorescent nanobeads, lateral flow assay, Escherichia coli O157:H7
\end{abstract}

\section{Short Communication}

Escherichia coli $\mathrm{O} 157: \mathrm{H} 7$ is an important serotype of enterohemorrhagic E. coli that can cause several clinical diseases, including diarrhea, hemorrhagic colitis, hemolytic uremic syndrome, and even death (Rabinovitz et al., 2012; Al-Nabulsi et al., 2016). The pathogenicity of E. coli O157:H7 is high: as little as 10 cfu can cause sickness (Ferens and Hovde, 2011). The main reservoir of $E$. coli is the gastrointestinal tract of cattle. In a previous survey conducted in 13 dairies, E. coli O157:H7

Received July 26, 2016.

Accepted August 12, 2016.

${ }^{1}$ Corresponding author: talktolaiwh@163.com was detected on 6 farms and in all farm elements, including milk filters (Toth et al., 2013). Poor hygienic and agricultural practices on farms, especially during milking, could lead to the contamination of milk and of dairy products prepared with raw milk (Miszczycha et al., 2016).

Conventional methods of isolating and identifying $E$. coli $\mathrm{O} 157$ :H7 from food samples are laborious and timeconsuming (Wang et al., 2015). Alternative methods such as ELISA (Amani et al., 2015), electrochemical methods (Wang et al., 2009), PCR (Wang et al., 2014), and quartz crystal microbalance sensing (Shen et al., 2011) are efficient, rapid, and sensitive. However, these methods are generally not suitable for onsite analysis because they require expensive instruments and complex operations. A simple, cost-effective, and rapid detection method for E. coli O157:H7 is needed.

The colloidal gold lateral flow assay is a rapid and simple method widely used in food safety detection (Peng et al., 2014), but its detection sensitivity is limited. The fluorescent nanobeads lateral flow (FNLF) assay is an excellent alternative to colloidal gold lateral flow, because its sensitivity for the detection of $E$. coli O157:H7 is better (Xie et al., 2014). Immunomagnetic separation (IMS) can rapidly enrich a target from complex matrices using immunomagnetic nanobeads (IMNB) in a magnetic field. Immunomagnetic separation significantly reduces detection time, improves sensitivity, and eliminates interference from fat, protein, and other food matrices (Shan et al., 2014).

In this study, we coupled IMS with FNLF to create an assay for the rapid detection of low-concentration $E$. coli O157:H7. As shown in Figure 1, IMNB were added to raw milk samples to enrich the target bacteria; target bacteria linked with the IMNB were eluted by heating; and then the eluent was detected using FNLF. To the best of our knowledge, this study is the first to combine IMS with FNLF for the rapid detection of pathogens.

We used the 1-(3-dimethylaminopropyl)-3-ethylcarbodiimide hydrochloride (EDC)-mediating method to 
prepare the IMNB (Wang et al., 2007, 2011). Carboxylated magnetic beads (1 mg; mean diameter $180 \mathrm{~nm}$; Allrunnano Technology Co. Ltd., Shanghai, China) were resuspended in $500 \mu \mathrm{L}$ of freshly prepared EDC $(20 \mathrm{mg} / \mathrm{mL}$ ) and $N$-hydroxysulfosuccinimide sodium salt (NHSS, $20 \mathrm{mg} / \mathrm{mL}$; Sigma Chemical Company, St. Louis, MO) in $0.01 M$ 2-morpholinoethanesulfonic acid buffer $(\mathrm{pH}$ 6.0) and then placed in a rotator (Ningbo Scientz Biotechnology Company, Ningbo, China) at 15 $\mathrm{rpm}$ for $15 \mathrm{~min}$. The solution was removed, and the magnetic beads were resuspended in $1 \mathrm{~mL}$ of borate saline buffer (0.01 $M, \mathrm{pH} 8.5)$ after incubation. Purified monoclonal antibodies $(50 \mu \mathrm{g}$; murine anti- $E$. coli O157: H7 monoclonal antibodies purchased from Meridian Life Science Inc., Memphis, TN) were immediately added to the solution with thorough mixing and then placed in a rotator at $15 \mathrm{rpm}$ for $4 \mathrm{~h}$. Antibody-conjugated magnetic beads were washed 3 times with PBS containing $0.05 \%$ Tween 20 . They were then blocked in $1 \mathrm{~mL}$ of PBS containing $1 \%$ BSA at room temperature for $1 \mathrm{~h}$, washed 4 times with PBS containing 0.05\% Tween 20, resuspended at a final concentration of $1 \mathrm{mg} / \mathrm{mL}$ in PBS, and then stored at $4^{\circ} \mathrm{C}$.

We mixed $1 \mathrm{~mL}$ of $E$. coli $\mathrm{O} 157: \mathrm{H} 7$ culture with 50 $\mu \mathrm{L}$ of IMNB $(0.05 \mathrm{mg})$. The mixture was incubated in a rotator at room temperature for $30 \mathrm{~min}$. The beads were washed twice with a magnetic separator (Zodolabs Biotech Co. Ltd., Wuxi, China). After the final wash, the supernatant was removed and the beads were resuspended in $100 \mu \mathrm{L}$ of PBS. The resuspended liquid was inactivated at $85^{\circ} \mathrm{C}$ for 15 min to elute the E. coli $\mathrm{O} 157$ : $\mathrm{H} 7$ for further detection.

We also used the EDC-mediating method to prepare the fluorescent nanobead (FN) probe (Xie et al., 2014). We mixed $10 \mathrm{~mL}$ of carboxylated FN (diameter $175 \mathrm{~nm}$, excitation wavelength $470 \mathrm{~nm}$, emission wavelength 525 $\mathrm{nm}, \mathrm{COOH}$ concentration $443 \mu \mathrm{Eq} / \mathrm{g}$; Merck, Darmstadt, Germany) with EDC and NHSS in 2-morpholinoethanesulfonic acid buffer saline ( $\mathrm{pH} 4.7)$ at room temperature for $120 \mathrm{~min}$ to form an amine-reactive NHSS ester. After adding $1 \mathrm{~mL}$ of anti-E. coli O157: H7 monoclonal antibodies (Meridian Life Science Inc.)

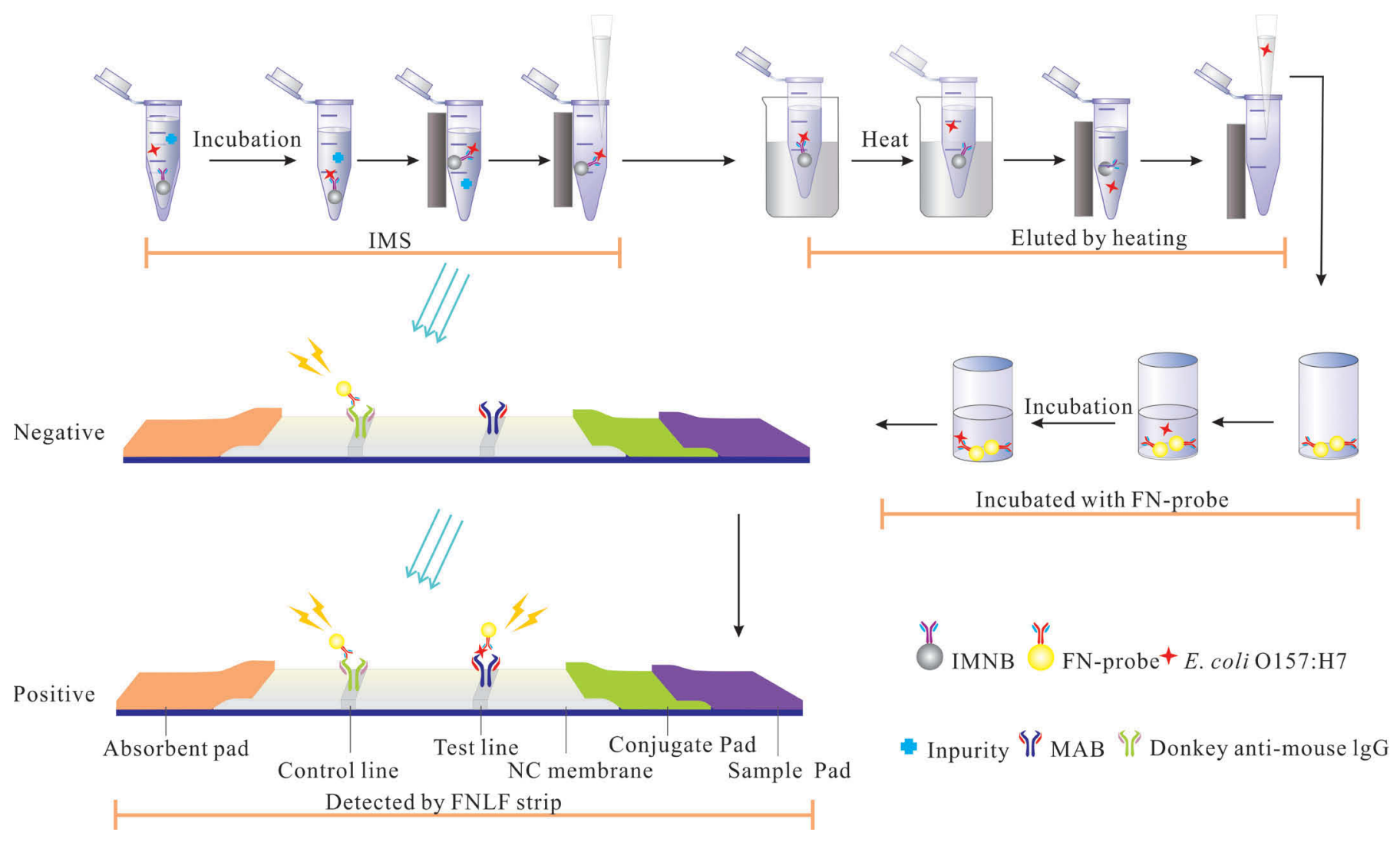

Figure 1. Schematic of immunomagnetic separation combined with fluorescent nanobeads lateral flow test strip in raw milk. IMS $=$ immunomagnetic separation; FN = fluorescent nanobeads; IMNB = immunomagnetic nanobeads; MAB = monoclonal antibody; FNLF $=$ fluorescent nanobeads lateral flow; $\mathrm{NC}=$ nitrocellulose. E. coli $=$ Escherichia coli. Color version available online. 
Table 1. Fluorescent signal intensity of the test line of fluorescent nanobeads lateral flow test strip with different concentrations of Escherichia coli $\mathrm{O} 157: \mathrm{H} 7$

\begin{tabular}{lcrc}
\hline $\begin{array}{l}\text { Concentration of } \\
\begin{array}{l}\text { E. coli } \text { O157:H7 } \\
(\mathrm{cfu} / \mathrm{mL})\end{array}\end{array}$ & $\begin{array}{c}\text { Mean of fluorescent } \\
\text { signal intensity }\end{array}$ & SD & $\mathrm{CV}(\%)$ \\
\hline $\mathrm{PBS}$ & 0 & - & - \\
$2.8 \times 10^{2}$ & 0 & - & - \\
$2.8 \times 10^{3}$ & 206 & 11 & 5.3 \\
$2.8 \times 10^{4}$ & 1,662 & 111 & 6.7 \\
$2.8 \times 10^{5}$ & 2,940 & 59 & 2 \\
$2.8 \times 10^{6}$ & 4,022 & 92 & 2.3 \\
\hline
\end{tabular}

at $50 \mu \mathrm{g} / \mathrm{mL}$, the solutions were incubated at room temperature for $2 \mathrm{~h}$. Afterward, $10 \%$ BSA was added to the mixture to block unreacted active sites of $\mathrm{FN}$ for 30 min. The reaction mixture was centrifuged at $8,000 \times g$ for $15 \mathrm{~min}$. Then, the pellet was suspended in $500 \mu \mathrm{L}$ of PBS (pH 7.0) containing 1\% BSA, 5\% sucrose, $3 \%$ trehalose, and $0.4 \%$ Tween 20 . The FN probe compound was dried on an ELISA plate at $30^{\circ} \mathrm{C}$ for $2 \mathrm{~h}$.

Escherichia coli O157:H7 polyclonal antibodies (1.5 $\mathrm{mg} / \mathrm{mL}$; Meridian Life Science Inc.) and donkey antimouse IgG secondary antibodies $(1.0 \mathrm{mg} / \mathrm{mL}$; Meridian Life Science Inc.) were diluted with PBS, spotted onto a nitrocellulose membrane to serve as the test line

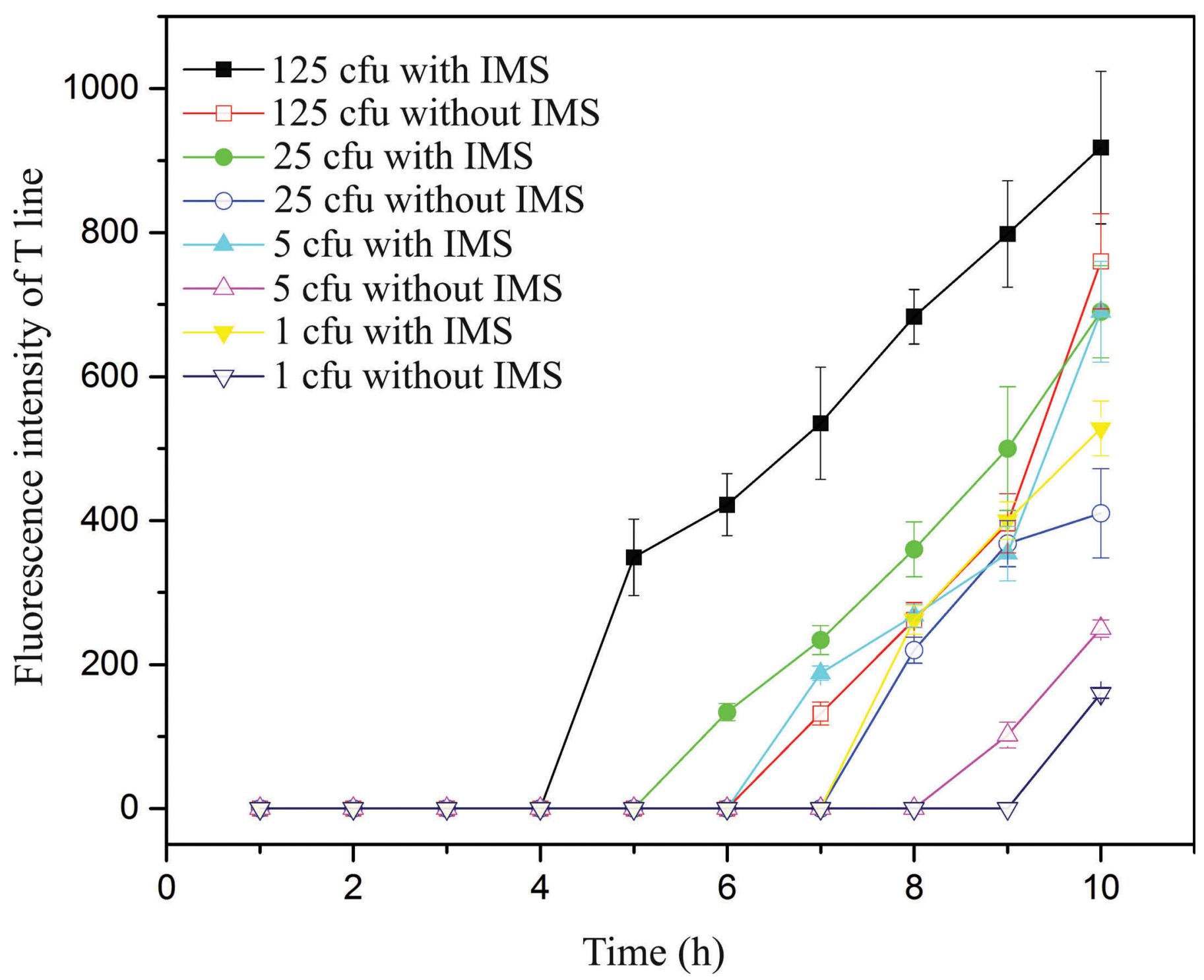

Figure 2. Fluorescence intensity of test (T) lines in detecting different inoculum levels of Escherichia coli O157:H7 with and without immunomagnetic separation (IMS). Error bars $=$ SD. Color version available online. 
and control line, and then vacuum dried for $2 \mathrm{~h}$. The nitrocellulose membrane, absorption pad, conjugate pad, and sample pad (Millipore, Bedford, MA) were assembled to create the lateral flow test strip (Figure 1).

One hundred microliters of PBS and E. coli O157:H7 serially diluted culture $\left(2.8 \times 10^{2}, 2.8 \times 10^{3}, 2.8 \times 10^{4}\right.$, $2.8 \times 10^{5}$, and $2.8 \times 10^{6} \mathrm{cfu} / \mathrm{mL}$ ) was dropped onto an ELISA plate containing the FN probe and left for 10 min to allow the antibody-antigen complex to form. Thereafter, samples were transmitted to the pads for the FNLF test strip. After 10 min, the FNLF test strip was examined using a fluorescent reader (excitation wavelength $470 \mathrm{~nm}$; Shanghai Huguo Science Instrument Co. Ltd., Shanghai, China). The signal value of the test line increased with the concentration of $E$. coli O157:H7. The sensitivity and CV of the FNLF test strip for E. coli O157: H7 assay were $2.8 \times 10^{3} \mathrm{cfu} / \mathrm{mL}$ and $5.3 \%$, respectively (Table 1 ).

In this study, we used $5 \mathrm{E}$. coli $\mathrm{O} 157: \mathrm{H} 7$ strains (CMCC 44828, ATCC 43888, NCTC 12900, XY0540, and XY0480) and 40 non-E. coli O157:H7 strains: Bacillus cereus (CMCC 63303, CMCC 63305, SLK), Bacillus licheniformis (CMCC 63519), Bacillus subtilis (BD366), Candida albicans (ATCC 10231, Z1), Enterobacter cloacae (CMCC 45301), Enterobacter sakazakii (CMCC 45401, CMCC 45402), enteropathogenic E. coli (CMCC 44496), E. coli (CMCC 44102, ATCC 25922), Lactobacillus bulgaricus (F1), Listeria grayi (ATCC 25401), Listeria innocua (ATCC 33090, ATCC 11288), Listeria ivanovii (ATCC 19119), Listeria monocytogenes (ATCC 13932, CMCC 54001, CMCC 54007), Listeria welshimeri serovar 6b (ATCC 35897), Listeria seeligeri (ATCC 35967), Micrococcus luteus (CMCC 28001), Proteus vulgaris (CMCC 49027), Pseudomonas aeruginosa (ATCC 27853, CMCC 10104), Salmonella anatum (ATCC 9270), Salmonella choleraesuis (ATCC 13312, CICC 21493), Salmonella enteritidis (ATCC 13076), Salmonella paratyphi A (ATCC 9150), Salmonella enterica (ATCC 10708), Salmonella typhimurium (ATCC 13311), Serratia marcescens (CMCC 41002), Shigella flexneri (ATCC 12022), Shigella sonnei (CMCC 51592), Staphylococcus aureus (CMCC 26001, CMCC 26003), and Vibrio parahaemolyticus (CGMCC 11997). All strains were obtained from Jiangxi Province Center for Disease Control and Prevention. The 5 E. coli O157:H7 strains $\left(10^{5} \mathrm{cfu} / \mathrm{mL}\right)$ presented positive signals with the FNLF test strip, and we observed no cross-reaction with the 40 non- $E$. coli $\mathrm{O} 157: \mathrm{H} 7$ strains $\left(10^{5} \mathrm{cfu} / \mathrm{mL}\right)$.

We inoculated raw milk ( $25 \mathrm{~mL})$ with $E$. coli O157:H7 $(1,5,25$, and $125 \mathrm{cfu})$. Each of the inoculated raw milk samples was added to $225 \mathrm{~mL}$ of sterile modified $E$. coli broth with novobiocin medium and incubated at $120 \mathrm{rpm}$ and $37^{\circ} \mathrm{C}$. A 1-mL aliquot of the enrichment culture was separated with IMNB and then evaluated using the FNLF test strip. We detected positive signals using the FNLF test strip after 10, 9, 8, and $7 \mathrm{~h}$ of enrichment in the modified $E$. coli broth with novobiocin medium. When the FNLF test strip was combined with IMS, the enrichment times were shortened to $8,7,6$, and $5 \mathrm{~h}$, respectively. The signal value of the FNLF test strip was stronger with IMS than without IMS (Figure 2), demonstrating that IMS can enrich E. coli O157:H7 and eliminate impurity interference.

This study demonstrated the feasibility of FNLF with IMS for the rapid, specific, and sensitive detection of $E$. coli O157:H7 in raw milk. The approach may be further extended to rapid detection of other food pathogens.

\section{ACKNOWLEDGMENTS}

We are grateful to the Jiangxi Agriculture Research System (JXARS-03), and the Jiangxi Education Bureau Technology (KJLD13009) for their financial support.

\section{REFERENCES}

Al-Nabulsi, A. A., A. N. Olaimat, T. M. Osaili, M. M. Ayyash, A. Abushelaibi, Z. W. Jaradat, R. Shaker, M. Al-Taani, and R. A. Holleyll. 2016. Behavior of Escherichia coli O157:H7 and Listeria monocytogenes during fermentation and storage of camel yogurt. J. Dairy Sci. 99:1802-1811. http://dx.doi.org/10.3168/jds.2015-9872.

Amani, J., A. Ahmadpour, A. A. I. Fooladi, and S. Nazarian. 2015. Detection of E. coli O157:H7 and Shigella dysenteriae toxins in clinical samples by PCR-ELISA. Braz. J. Infect. Dis. 19:278-284.

Ferens, W. A., and C. J. Hovde. 2011. Escherichia coli O157:H7: Animal reservoir and sources of human infection. Foodborne Pathog. Dis. 8:465-487.

Miszczycha, S. D., N. Bel, P. Gay-Perret, V. Michel, M. C. Montel, and D. Sergentet-Thevenot. 2016. Short communication: Behavior of different Shiga toxin-producing Escherichia coli serotypes (O26:H11, O103:H2, O145:H28, O157:H7) during the manufacture, ripening, and storage of a white mold cheese. J. Dairy Sci. 99:5224-5229. http://dx.doi.org/10.3168/jds.2015-10803.

Peng, T., W. C. Yang, W. H. Lai, Y. H. Xiong, H. Wei, and J. Zhang. 2014. Improvement of the stability of immunochromatographic assay for the quantitative detection of clenbuterol in swine urine. Anal. Methods 6:7394-7398.

Rabinovitz, B. C., E. Gerhardt, C. Tironi Farinati, A. Abdala, R Galarza, D. A. Vilte, C. Ibarra, A. Cataldi, and E. C. Mercado. 2012. Vaccination of pregnant cows with EspA, EspB, gammaintimin, and Shiga toxin 2 proteins from Escherichia coli O157:H7 induces high levels of specific colostral antibodies that are transferred to newborn calves. J. Dairy Sci. 95:3318-3326. http:// dx.doi.org/10.3168/jds.2011-5093.

Shan, S., Z. Zhong, W. Lai, Y. Xiong, X. Cui, and D. Liu. 2014. Immunomagnetic nanobeads based on a streptavidin-biotin system for the highly efficient and specific separation of Listeria monocytogenes. Food Contr. 45:138-142.

Shen, Z. Q., J.-F. Wang, Z. G. Qiu, M. Jin, X. W. Wang, Z. L. Chen, J. W. Li, and F. H. Cao. 2011. QCM immunosensor detection of Escherichia coli O157:H7 based on beacon immunomagnetic nanoparticles and catalytic growth of colloidal gold. Biosens. Bioelectron. 26:3376-3381.

Toth, J. D., H. W. Aceto, S. C. Rankin, and Z. Dou. 2013. Short communication: Survey of animal-borne pathogens in the farm 
environment of 13 dairy operations. J. Dairy Sci. 96:5756-5761. http://dx.doi.org/10.3168/jds.2012-6499.

Wang, J. Y., M. H. Chen, Z. C. Sheng, D. F. Liu, S. S. Wu, and W. H. Lai. 2015. Development of colloidal gold immunochromatographic signal-amplifying system for ultrasensitive detection of Escherichia coli O157:H7 in milk. RSC Advances 5:62300-62305.

Wang, L., P. Li, Z. Zhang, Q. Chen, Z. P. Aguilar, H. Xu, L. Yang, F. $\mathrm{Xu}$, W. Lai, and Y. Xiong. 2014. Rapid and accurate detection of viable Escherichia coli O157: $\mathrm{H} 7$ in milk using a combined IMS, sodium deoxycholate, PMA and real-time quantitative PCR process. Food Contr. 36:119-125.

Wang, L., Q. Liu, Z. Hu, Y. Zhang, C. Wu, M. Yang, and P. Wang. 2009. A novel electrochemical biosensor based on dynamic poly- merase-extending hybridization for E. coli O157:H7 DNA detection. Talanta 78:647-652.

Wang, L., W. Zhao, M. B. O'Donoghue, and W. Tan. 2007. Fluorescent nanoparticles for multiplexed bacteria monitoring. Bioconjug. Chem. 18:297-301

Wang, Y., Y. Bai, and X. Wei. 2011. Conjugation behaviours of CdTe quantum dots and antibody by a novel immunochromatographic method. IET Nanobiotechnology 5:14-19.

Xie, Q. Y., Y. H. Wu, Q. R. Xiong, H. Y. Xu, Y. H. Xiong, K. Liu, Y. Jin, and W. H. Lai. 2014. Advantages of fluorescent microspheres compared with colloidal gold as a label in immunochromatographic lateral flow assays. Biosens. Bioelectron. 54:262-265. 